\title{
Interactive comment on "Measurement and modelling of rainfall partitioning by deciduous Potentilla fruticosa shrub on the Qinghai-Tibet Plateau, China” by Si-Yi Zhang and Xiao-Yan Li
}

\author{
J. Van Stan (Referee) \\ jvanstan@georgiasouthern.edu \\ Received and published: 17 December 2016
}

Manuscript \#2016-589 by Zhang and Li examines/models rainfall partitioning of a shrub species in an alpine semiarid site (Qinghai Lake, China). Although there are some interesting aspects (i.e., directly measured variable canopy structural parameters in the model), my opinion is that this study does not require publication in an international top-tier journal outlet. Thus, I recommend rejection from HESS. Rather, I believe this study is better suited for a journal outlet focused on the region within which it is situated as substantial (and excellent) work has already been published on the precipitation partitioning of plant canopies in this area (the authors' works cited section includes many examples). Yes, this study adds one more shrub species to the list of plants 
studied (with some details on canopy structural variability), but is that broad enough?

Besides this issue, I have other concerns:

1) Methods are missing details. Specifically...

${ }^{* *}$ Was only 1 rainfall gauge used? For the past several years, rainfall measurement protocols have necessitated three rainfall gauges (i.e., see International Co-operative Programme - ICP Forests). If only 1 rainfall gauge was used, please justify and acknowledge the difference between this study and current standard rainfall measurement protocols.

${ }^{* *}$ How were the few throughfall gauges distributed in the patches? Seeing as very few throughfall gauges were deployed (see point 2) and throughfall is spatially heterogeneous, knowledge of the arrangement of gauges is necessary to provide the reader an idea of how well represented the spatial heterogeneity was in the study's observations. Since there were so few gauges, were they roved around? Other concerns about throughfall observations shared later (see point 2)

${ }^{* *}$ What were the dimensions of the stemflow collection devices? It is mentioned that "sinks" of aluminum foil (P6, L12) were used to collect stemflow. How big was the sink area? Sinks that extend far from the shrub stem may be gathering throughfall as well as stemflow. This might explain stemflow accounting for nearly $30 \%$ of gross rainfall $-a$ rather high, albeit possible, proportion. Knowing the dimensions of the stemflow "sink" will strengthen (or weaken) confidence in the quite large stemflow production. Also, how long after a storm event were stemflow gauges manually measured? If stemflow measurements were not taken immediately after a storm, were there efforts to minimize evaporation losses from the collectors? Other concerns about stemflow observations shared later (see point 3)

2) Throughfall variability may have been too under-sampled. There were very few throughfall collectors ( $n=9$ total, $n=3$ per plot: P7, L11-14) each with a small col-

Printer-friendly version

Discussion paper 
lection area (3.34 cm diameter: P76, L12), which likely prevents accurate throughfall estimation considering the well-documented spatial variability of throughfall.

3) Stemflow estimates may not be representative due to selective sampling. Stemflow observations were selected from very few storms $(n=8$ : P6, L16). Did these storms represent the continuum of storm magnitudes and intensities generally experienced at the site? If not, meteorological conditions that favor stemflow generation may explain the high stemflow proportion. Stemflow observations were also selected from very few stems ( $n=6: P 6, L 17)$. Where were these stems located in the patches? Were they on the edge or interior to the shrub patch? Were the patched trimmed to install stemflow collars (which may create an artificial edge effect)? This is important to know as location within the patch can affect stemflow generation. Also, how did the selected stems, and canopy draining to those stems, compare to the range of canopy characteristics at the site?

4) I have some minor concerns with the edits to the reformulated Gash model in this study. I say "minor" because it is regarding only 2 assumptions that simplified evaporation estimates:

(a) The authors' assume that "shrub canopy evaporation has no difference from the stem evaporation" (P10, L18 - P11, L1) but provide no data in support of the assumption. Without data supporting the authors' claim, I'm inclined to believe that stem and canopy evaporation rates would be different due to reasons commonly identified in past literature: (i) canopy shading the stem, (ii) different albedo of leaf and stem surfaces, (iii) wind speeds being reduced from canopy edge to the interior stem, and (iv) complex stem bark surfaces (like shown in Fig. 3b) may shelter entrained water from meteorological conditions driving evaporation.

(b) The authors' also assume that "evaporation from the canopy and from the ground is equal, because the height of the shrub is only about $35 \mathrm{~cm}$ "; however, the physical drivers of evaporation can differ between the ground and shrub canopy despite modest

Printer-friendly version

Discussion paper 
differences in elevation.

5) The manuscript is in need of significant English language editing. As it would take too much time to identify and suggest changes for all of the necessary language editing, an example in each section are provided to guide the authors during their revisions:

Abstract, P1, L10- "has not get enough attention" should be "has not gotten enough attention". . . but, my opinion is that the language shouldn't be so colloquial. It would be better to state something like "has not received enough attention"

Introduction P2, L11- "The gross precipitation reaches the canopy..." should be "The gross precipitation that reaches the canopy..." and the authors incorrectly state that the canopy partitions precipitation only into interception, stemflow and "free" throughfall. This ignores all "release" throughfall produced from canopy contact. Why not just say "interception stemflow and throughfall" as the general term "throughfall" implies the sum of free and release throughfall?

Methods P6, L7- "rainfall events were discretized by assuming without rainfall between events of $12 \mathrm{~h}$..." should be something like "rainfall events were discretized by assuming a minimum inter-event time of $12 \mathrm{~h}$..." Minimum inter-event time is a common term in precipitation partitioning literature (i.e., Dunkerley, 2015, Hydrol Process, 29, 3294 and Llorens et al., 2014, J Hydrol, 512, 254). I would also recommend the authors' follow the convention for introducing species: latin name (taxonomic authority, common name). Thereafter, the use of the abbreviated latin name is typically used.

Results P15, L13 - I think the line "which occupying 14.4\% of the total observed interception" should be "which accounted for $14.4 \%$ of the total observed interception"

Discussion P17, L11-12 - "The proportion pt also has important in the stemflow of course" needs to be rewritten for clarity as I'm unsure what the authors are saying.

Conclusion P26, L9 to P27, L2 - this statement is unclear. Does "available water that free fell and drained along the stem" mean "stemflow"? Why would "free" falling

Printer-friendly version

Discussion paper 
droplets "drain along the stem"? Or, does this statement simply mean "throughfall and stemflow"? Please revise for clarity.

Interactive comment on Hydrol. Earth Syst. Sci. Discuss., doi:10.5194/hess-2016-589, 2016. 